ARTIGO ORIGINAL ORIGINAL ARTICLE

\title{
Análise de custo-efetividade da toxina onabotulínica A no tratamento preventivo da migrânea crônica sob a perspectiva do sistema privado de saúde do Brasil
}

\author{
Cost-effectiveness analysis of onabotulinumtoxinA in \\ the preventive treatment of chronic migraine under the \\ Brazilian private health care system perspective \\ Elcio Juliato Piovesan ${ }^{1}$, Patricia Cadecaro², Camila Pepe ${ }^{3}$ \\ DOI: 10.21115/JBES.v9.suppl1.71-80
}

Palavras-chave:

cefaleia, hemicrania, análise custo-benefício, toxina botulínica A, toxina onabotulínica $A$

\section{Keywords:}

headache, migraine, cost-benefit analysis, onabotulinumtoxinA, botulinum toxin type $\mathrm{A}$

\begin{abstract}
RESUMO
Objetivo: Avaliar o custo-efetividade da toxina onabotulínica A (TB) versus ausência de tratamento "não tratar" (NT) da migrânea crônica refratária a pelo menos dois medicamentos em um Sistema de Saúde Suplementar. Métodos: Modelo analítico de decisão simulou desfechos e custos em 24 semanas. Os desfechos clínicos foram: dias com cefaleia (DC); dias com cefaleia moderada/severa (DCMS); episódios de cefaleia (EC); dias com migrânea (DM) e episódios de migrânea (EM). Para o braço NT, utilizaram-se os valores basais do placebo. Para o braço TB, utilizaram-se os valores basais aplicando as reduções relacionadas ao uso do tratamento. Os custos incluídos foram custos médicos diretos. Os dados que subsidiaram as análises foram extraídos da literatura. Avaliou-se uma aplicação de TB (155U) a cada 12 semanas, totalizando duas aplicações em 24 semanas. Custos foram extraídos de bases oficiais do Brasil. Considerou-se taxa de descontinuação de 3,8\% para TB na semana 24. Análises de sensibilidade probabilística (ASP) e univariada (ASU) foram realizadas para o desfecho DM. Resultados: $O$ uso de TB proporcionou redução de 52,42\% nos DC; 47,97\% nos DCMS; 37,60\% nos EC; 50,49\% nos DM; 35,68\% nos EM e uma redução de R\$1.193 com consulta médica e hospitalização. Houve incremento de R\$ 8.646 com aplicação da TB, resultando numa Razão de Custo-Efetividade Incremental (RCEI) de R\$ 142,16/DC; R\$ 155,35/DCMS; R\$ 198,22/EC; R\$ 147,61/ DM e R\$208,85/EM. A ASP e ASU comprovaram os resultados do caso base. Conclusão: Uso de TB proporciona redução dos desfechos clínicos, variando de 44\% a 49\%, além de redução de 54\% a 60\% nos custos com visitas médicas e hospitalização.
\end{abstract}


plications in 24 weeks. Costs were extracted from Brazilian official databases. A 3.8\% discontinuation rate was considered for OT at week 24. Probabilistic (PSA) and univariate (USA) sensitivity analyzes were performed for the DM outcome. Results: The use of OT provided a reduction of $52.42 \%$ in DH; 47.97\% in DMSH; $37.60 \%$ in HE; 50.49\% in DM; 35.68\% in EM and a reduction of $\mathrm{R} \$ 1,193$ with medical visits and hospitalization. There was an increase of $\mathrm{R} \$ 8,646$ with OT application; resulting in an incremental cost-effectiveness ratio (ICER) of R\$ 142.16/DH; R\$ 155.35/DMSH; R\$ 198.22/HE; R\$ 147.61/ DM and R\$208.85/EM. PSA and USA proved the base case results. Conclusion: Use of OT provides a reduction in clinical outcomes ranging from $44 \%$ to $49 \%$, as well as a reduction of $54 \%$ to $60 \%$ in costs with medical visits and hospitalization.

\section{Introdução}

A migrânea, ou enxaqueca, é um distúrbio neurológico debilitante, caracterizada por episódios recorrentes de cefaleia, geralmente de aspecto unilateral, pulsátil de moderada ou severa intensidade (Robertson \& Garza, 2012). Sua etiologia não é completamente conhecida, contudo, sabe-se que os episódios de migrânea se caracterizam por uma combinação de alterações neurovasculares, que podem ser desencadeadas por fatores internos e externos como, por exemplo, estresse, jejum, privação de sono, ingestão de álcool, consumo excessivo de cafeína e fatores hormonais (Krymchantowski et al., 1999). Os pacientes que apresentam migrânea possuem maior probabilidade de desenvolver outras doenças como asma, depressão, acidentes cerebrovasculares e epilepsia (Bigal et al., 2000). Adicionalmente, a cefaleia da migrânea pode ser desencadeada e posteriormente agravada por atividade física, presença de náuseas e/ou vômitos, fotofobia e fonofobia (Headache Classification Committee of the International Headache Society - IHS, n.d).

De acordo com os dados da Organização Mundial da Saúde (OMS) (Word Health Organization, 2011), a cefaleia representa um dos motivos mais frequentes de consultas médicas, constando-se a migrânea entre as vinte doenças mais incapacitantes durante a vida de um indivíduo. Essa modalidade de cefaleia apresenta prevalência anual entre 3\% e 27,5\% (Stovner et al., 2007). Na América Latina, o sexo masculino é afetado entre 2,9\% e 7,8\% da população, e o feminino, entre 10,1\% e 17,4\% (Giacomozzi et al., 2012).

Conforme o padrão semiológico da cefaleia, a migrânea pode ser subdividida em diferentes subtipos. A subforma migrânea crônica apresenta como seu epicentro diagnóstico um aumento na frequência das crises de cefaleia, sendo essa uma entidade relativamente comum. Estudos populacionais mundiais mostram que a prevalência dela oscila entre 0,9\% e 5,1\% (Natoli et al., 2010; Lipton et al., 2007). O estudo epidemiológico sobre a migrânea crônica no Brasil aponta a prevalência de 5,12\% (Giacomozzi et al., 2012).

Clinicamente, caracteriza-se pela presença da cefaleia ocorrendo em 15 ou mais dias no mês, durante um período mínimo de três meses (não necessariamente contínuos, mas dentro de um prazo de doze meses). Destes 15 dias, em pelo menos oito deles a cefaleia deve apresentar características de migrânea, ou seja, no mínimo duas dos padrões a seguir (1. Dor com localização unilateral; 2. Dor pulsátil; 3. Dor moderada a severa intensidade; 4. Dor que agrava durante atividades físicas rotineiras) e no mínimo um dos padrões a seguir (1. Náuseas e ou vômitos; 2. Fotofobia e fonofobia) (Headache Classification Committee of the International Headache Society - IHS, n.d.).

De acordo com o Consenso Latino-Americano para as Diretrizes de Tratamento da Migrânea Crônica de 2012, o objetivo do tratamento é reduzir a frequência, a duração e a intensidade das crises e melhorar sua resposta ao tratamento agudo, diminuindo, dessa maneira, seu impacto na qualidade de vida do paciente. A adequada abordagem da migrânea crônica deve estabelecer estratégias de tratamento de acordo com sua fase evolutiva. Estabelecem-se como delineamento as fases: aguda (controle imediato da dor); fase de transição de migrânea crônica até a forma episódica (Fase de dessensibilização) e fase preventiva de controle da forma episódica para evitar evolução para a forma crônica novamente (Giacomozzi et al., 2012).

A toxina onabotulínica $A\left(\right.$ Botox $\left.^{\circledast}\right)$, também conhecida como onabotulinumtoxinA, é uma potente neurotoxina produzida pela bactéria Gram-positiva Clostridium botulinum. (Linsenmeyer, 2013). O processo presumido para o mecanismo de dessensibilização da migrânea crônica é o bloqueio de sinais periféricos para o sistema nervoso central, que inibe e reverte a sensibilização central, conforme confirmado em estudos pré-clínicos e clínicos. A toxina bloqueia a liberação de neurotransmissores associados com a origem da dor (Allergan, 2015).

O uso da toxina onabotulínica A para o tratamento da migrânea crônica é registrado desde 2010 nos Estados Unidos e 2011 na Grã-Bretanha e na União Europeia, sendo esta indicada para o tratamento das migrâneas crônicas e refratárias com comprometimento importante da qualidade de vida e das atividades laborativas, sociais, familiares e de lazer (Allergan, 2015; Bumb et al., 2013). No Brasil, sua utilização é aprovada pela Anvisa/MS. Destina-se ao tratamento de pacientes com migrânea crônica, entre outras indicações (Frampton, 2012). Sua eficácia e sua segurança na migrânea crônica foram demonstradas em ensaios clínicos e metanálises (Aurora et al., 2010; Aurora et al., 2011; Diener et al., 2010; Dodick et al., 2010; Jackson et al., 2012; Matthew et al., 2005; Rothrock et 
al., 2014; Silberstein et al., 2005). As mais recentes recomendações da Academia Americana de Neurologia citam que a toxina onabotulínica A é estabelecida como efetiva e deve ser oferecida para aumentar os dias livres de cefaleia (com nível A de evidência) e deve ser considerada para aumentar a qualidade de vida relacionada à saúde em pacientes com migrânea crônica (com nível B de evidência) (American Academy of Neurology, 2016).

Do ponto de vista financeiro, a migrânea crônica impõe um significativo impacto na economia, gerando altos custos de maneira direta e indireta para os pacientes e sociedade, com comprometimento da qualidade de vida dos portadores. Além disso, é uma das principais causas de absenteísmo e diminuição da produtividade no trabalho. (Krymchantowski et al., 1999). A migrânea crônica refratária (ausência de resposta terapêutica há pelo menos duas classes de medicamentos utilizados com intuito de reverter a sensibilização nociceptiva central) parece ser o cenário clínico mais dramático dessa entidade. Neste caso, com o uso da toxina onabotulínica A houve $55 \%$ de redução nas visitas a serviços de emergência, 57\% menos hospitalizações e uma redução de $49,7 \%$ no custo total durante os seis meses de tratamento, em comparação ao período pré-tratamento (Rothrock et al., 2014).

Atualmente, o tratamento utilizando toxina onabotulínica A não está disponível no sistema de saúde privado no Brasil e os pacientes refratários a tratamentos medicamentosos permanecem sem opção terapêutica, com ônus no estado de saúde e na qualidade de vida, além do impacto econômico para o sistema, com episódios recorrentes. Em um contexto em que ainda existem necessidades médicas não atendidas, este estudo teve como objetivo avaliar o perfil de custo-efetividade da toxina onabotulínica A no tratamento de pacientes com migrânea crônica sob a perspectiva do Sistema de Saúde Suplementar (SSS) no Brasil.

\section{Métodos}

\section{Estrutura do modelo de custo-efetividade}

O tipo de análise selecionada foi a análise de custo-efetividade, uma vez que o modelo tem o objetivo de comparar os custos médicos diretos e os desfechos de saúde envolvidos no tratamento de pacientes com migrânea crônica utilizando toxina onabotulínica A versus o não tratamento.

Avaliou-se o horizonte temporal de 24 semanas, definido de acordo com a duração da fase duplo-cego da análise agrupada dos estudos PREEMPT (Dodick et al., 2010). A análise também foi extrapolada para os horizontes temporais de 48 e 96 semanas.

Foi aplicada uma taxa de desconto anual de 5\% para custos e desfechos, de acordo com as recomendações das Diretrizes Metodológicas para Estudos de Avaliação Econô- mica de Tecnologias em Saúde, publicadas pelo Ministério da Saúde (Ministério da Saúde, Brasil. Secretaria de Ciência, Tecnologia e Insumos Estratégicos, Departamento de Ciência e Tecnologia, 2009).

A tecnologia avaliada neste estudo foi o uso da toxina onabotulínica A e o comparador considerado para a análise foi o não tratamento ("sem tratamento").

Desenvolveu-se um modelo analítico no software Microsoft Excel ${ }^{\circledR} 2013$ para simular os desfechos e custos associados ao tratamento da migrânea crônica.

Para o comparador ("sem tratamento"), utilizaram-se os valores basais do placebo para contabilizar os dias ou episódios de migrânea ou cefaleia. Como nenhuma intervenção foi utilizada neste braço, não foram aplicadas reduções nas ocorrências dos desfechos.

Para a toxina onabotulínica A, partiu-se dos valores basais e aplicaram-se as reduções de desfechos referentes ao tratamento para mensurar o benefício clínico do tratamento nesta população.

Para os horizontes maiores que 24 semanas, extrapolaram-se os dados de forma linear.

Os resultados comparativos das estratégias alternativas de tratamento foram medidos pela razão de custo-efetividade incremental (RCEI). Esta é definida, para duas alternativas de tratamento específicos, como o custo adicional proporcionado pelo tratamento em análise dividido pelo ganho adicional em saúde alcançado pelo mesmo.

Os desfechos de saúde considerados foram: (i) Dias com cefaleia; (ii) Dias com cefaleia moderada/severa; (iii) Episódios de cefaleia; (iv) Dias com migrânea; (v) Episódios de migrânea.

Os desfechos econômicos contemplados foram custos médicos diretos, incluindo os recursos médicos utilizados diretamente para o tratamento do paciente, como custos dos medicamentos, consultas de acompanhamento, exames, procedimentos médicos e hospitalizações.

Portanto, uma RCEI foi calculada indicando o total de recursos necessários para que se evite um dia com cefaleia, um dia com cefaleia moderada/grave, um episódio de cefaleia, um dia com migrânea ou um episódio de migrânea, no horizonte de tempo analisado.

A RCEl foi calculada da seguinte forma:

RCEI = CUSTO INCREMENTAL / EFETIVIDADE INCREMENTAL

Onde:

- Custo incremental = Custo com toxina onabotulínica

A - Custo com o não tratamento

- $\quad$ Efetividade incremental $=\left(N^{\circ}\right.$ de dia com cefaleia* com toxina onabotulínica $\mathrm{A}-\mathrm{N}^{\circ}$ de dia com cefaleia* com não tratamento)

* De acordo com o desfecho de saúde analisado: (i) Dias com cefaleia; (ii) Dias com cefaleia moderada/severa; (iii) Episódios de cefaleia; (iv) Dias com migrânea; (v) Episódios de migrânea. 


\section{Dados de eficácia}

Os parâmetros de eficácia foram obtidos a partir do estudo de Dodick, et al., 2010, uma análise agrupada dos resultados dos estudos PREEMPT, apresentando os valores basais para os desfechos e as mudanças deles após o tratamento (Dodick et al., 2010).

A Tabela 1 apresenta a ocorrência em dias e de episódios de cefaleia e migrânea no início do estudo e a Tabela 2 apresenta a redução deles após 24 semanas de tratamento. Exceto para episódios de cefaleia ( $p$-valor $=0,009)$, para os demais desfechos foram apresentadas diferenças significativas a favor da toxina onabotulínica A (Dodick et al., 2010).

Tabela 1. Características do baseline

\begin{tabular}{|c|c|c|c|}
\hline Desfecho & $\begin{array}{c}\text { Toxina } \\
\text { onabotulínica } \\
A(d p)\end{array}$ & Placebo (dp) & P-valor \\
\hline Dias com cefaleia & $19,9( \pm 3,68)$ & $19,8(+3,68)$ & 0,498 \\
\hline $\begin{array}{l}\text { Dias com cefaleia } \\
\text { moderada/severa }\end{array}$ & $18,1(+4,12)$ & $18,0(+4,25)$ & 0,705 \\
\hline Episódios de cefaleia & $12,2(+5,25)$ & $13,0(+5,5)$ & 0,004 \\
\hline Dias com migrânea & $19,1(+3,99)$ & $18,9(+4,05)$ & 0,328 \\
\hline Episódios de migrânea & $11,4(+5,02)$ & $12,2(+5,42)$ & 0,004 \\
\hline
\end{tabular}

As comparações entre os grupos de tratamento foram feitas por análise de covariância. Para as variáveis binomiais, as comparações entre grupos foram realizadas com o teste qui-quadrado de Pearson ou com o teste exato de Fisher. Diferenças estatisticamente significativas $p<0,05$.

\section{Dados de custo}

\section{Utilização de recursos em saúde}

O estudo econômico de mundo real de Rothrock et al., 2014, que avalia a redução de utilização de recursos e custos associados após o tratamento da migrânea crônica com a toxina onabotulínica A, foi utilizado para alimentar o modelo econômico com dados de utilização de recurso (Rothrock et al., 2014). O estudo apresenta o número médio de visitas médicas, visitas a emergência e hospitalizações pré- e pós-tratamento com toxina onabotulínica A (Rothrock et al., 2014). Para o placebo, assumiram-se os valores médios de uso de recurso dos pacientes antes do tratamento com toxina onabotulínica A (Tabela 3).

\section{Custo de aquisição e administração da toxina onabotulínica $A$}

Os custos de aquisição de medicamentos foram obtidos por meio de preços de lista publicados pela Câmara de Regulação do Mercado de Medicamentos (CMED), considerando o preço fábrica (PF) com 18\% de ICMS, conforme a atualização de novembro de 2016, de R\$3.238,32 para a apresentação de $200 \mathrm{U}$ (Agência Nacional de Vigilância Sanitária - Anvisa; Câmara de Regulação do Mercado de Medicamentos - CMED, 2016).

Assumiu-se o custo de administração da toxina onabotulínica A igual a quatro vezes o custo de bloqueio fenólico, alcoólico ou com toxina onabotulínica por segmento corporal (CBHPM 2.01.03.14-0), sendo o custo total de administração igual a R\$ 1.085,45 (AMB - Associação Médica Brasileira, 2015).

Tabela 2. Mudança do baseline após 24 semanas

\begin{tabular}{lccc}
\hline Desfecho & Toxina onabotulínica A & Diferença de média (IC 95\%) & P-valor \\
\hline Dias com cefaleia & $-8,4$ & $-1,8(-2,52 ;-1,13)$ & $<0,001$ \\
\hline $\begin{array}{l}\text { Dias com cefaleia } \\
\text { moderada/severa }\end{array}$ & $-7,7$ & $-1,9(-2,62 ;-1,26)$ & $<0,001$ \\
\hline Episódios de cefaleia & $-5,2$ & $-0,3(-1,17 ;-0,17)$ & 0,009 \\
\hline Dias com migrânea & $-8,2$ & $-2,0(-2,67 ;-1,27)$ & $<0,001$ \\
\hline Episódios de migrânea & $-4,9$ & $-0,4(-1,20 ;-0,23)$ & 0,004 \\
\hline
\end{tabular}

As comparações entre os grupos de tratamento foram feitas por análise de covariância. Para as variáveis binomiais, as comparações entre grupos foram realizadas com o teste qui-quadrado de Pearson ou com o teste exato de Fisher.

Diferenças estatisticamente significativas $p<0,05$.

Tabela 3. Utilização de recursos pré- e pós-tratamento

\begin{tabular}{lcccc}
\hline Recurso & $\begin{array}{c}\text { Pré-tratamento } \\
\text { (Placebo) }\end{array}$ & $\begin{array}{c}\text { Pós-tratamento } \\
\text { (Toxina onabotulínica A) }\end{array}$ & Diferença de média & P-valor \\
\hline Consulta médica & 1,67 & 0,76 & $-0,92 \pm 2,04$ & $<0,001$ \\
\hline Visita à emergência & 0,65 & 0,27 & $-0,39 \pm 1,46$ & $<0,001$ \\
\hline Hospitalização & 0,20 & 0,08 & $-0,11 \pm 0,55$ & 0,002 \\
\hline
\end{tabular}

As comparações entre os grupos de tratamento foram feitas por análise de covariância. Para as variáveis binomiais, as comparações entre grupos foram realizadas com o teste qui-quadrado de Pearson ou com o teste exato de Fisher.

Diferenças estatisticamente significativas $p<0,05$. 
Para o custo de tratamento, foram consideradas duas aplicações a cada 24 semanas (aproximadamente seis meses), sendo definido um limite de quatro aplicações por ano, assumindo que foi consumido um frasco de $200 \mathrm{U}$ de toxina onabotulínica A por administração.

Para a composição de custo de tratamento, foram consideradas taxas de descontinuação de 3,8\% e 0\% para os pacientes em tratamento com toxina onabotulínica A e com "sem tratamento", respectivamente. Apesar de o estudo PREEMPT apresentar uma taxa de descontinuação de 1,2\% para o placebo, uma vez que seu tratamento não implica alterações no custo, optou-se por considerá-la nula no modelo (Dodick et al., 2010). Também se considerou que a descontinuação ocorre na semana 24, sendo assim, mesmo descontinuado, o paciente implicará o custo de duas aplicações de toxina onabotulínica A.

O custo de tratamento para 24, 48 e 96 semanas está sintetizado na Tabela 4.

Tabela 4. Custo de tratamento com toxina onabotulínica A

\begin{tabular}{lccc}
\hline & 24 semanas & 48 semanas & 96 semanas \\
\hline $\begin{array}{l}\text { Tratamento } \\
\text { contínuo (96,2\%) }\end{array}$ & $\mathrm{R} \$$ 8.317,01 & $\mathrm{R} \$$ 16.634,03 & $\mathrm{R} \$ 33.268,05$ \\
\hline $\mathrm{N}^{\circ}$ de aplicações & 2 & 4 & 8 \\
\hline $\begin{array}{l}\text { Descontinuação } \\
(3,8 \%)\end{array}$ & $\mathrm{R} \$ 328,53$ & $\mathrm{R} \$ 328,53$ & $\mathrm{R} \$ 328,53$ \\
\hline $\begin{array}{l}\text { Doses até } \\
\text { descontinuação }\end{array}$ & 2 doses & 2 doses & 2 doses \\
\hline Custo total & $\mathrm{R} \$$ 8.645,54 & $\mathrm{R} \$ 16.962,56$ & $\mathrm{R} \$ 33.596,58$ \\
\hline
\end{tabular}

\section{Custo de acompanhamento do paciente}

A Tabela 5 apresenta o custo de consulta médica, visita à emergência e hospitalização obtidos por meio da utilização de recurso apresentada pelo estudo de Rothrock et al., 2014, com base nos custos apresentados na $5^{a}$ edição - Classificação Brasileira Hierarquizada de Procedimentos Médicos (CBHPM) (Agência Nacional de Vigilância Sanitária - Anvisa; Câmara de Regulação do Mercado de Medicamentos - CMED, 2016; Associação Médica Brasileira - AMB, 2015; Rothrock et al., 2014).

Tabela 5. Custos médicos para ambos os grupos de tratamento

\begin{tabular}{lc}
\hline Custo & Valor \\
\hline Consulta médica & $R \$ 309,65$ \\
\hline Visita à emergência & $R \$ 1.048,03$ \\
\hline Hospitalização & $R \$ 4.277,36$ \\
\hline
\end{tabular}

\section{Análise de sensibilidade probabilística}

A análise de sensibilidade probabilística considera variações de diversos parâmetros por vez e foi realizada por meio da atribuição de uma distribuição de probabilidade apropriada para cada um dos parâmetros analisados. Para os parâmetros de custo, foi atribuída a distribuição de probabilidade gama, para as quantidades de eventos (por exemplo, número de visitas médicas) foi atribuída uma distribuição log-normal e para as probabilidades foi atribuída a distribuição beta. A análise foi calculada com 1.000 interações. Os resultados foram avaliados e classificados em: Quadrante 1 (efetividade incremental > 0 e custo incremental > 0); Quadrante 2 (efetividade incremental $<0$ e custo incremental $>0$ ); Quadrante 3 (efetividade incremental $<0$ e custo incremental $<0$ ) e Quadrante 4 (efetividade incremental $>0$ e custo incremental $<0$ ).

\section{Análise de sensibilidade univariada}

Análises de sensibilidade univariadas consideram variações de um único parâmetro por vez, mantendo os demais parâmetros constantes. Neste caso, os parâmetros considerados críticos foram variados a partir do seu valor no cenário base para valores limite, e os resultados obtidos para o custo por um dia de cefaleia evitado foram documentados para avaliar a robustez dos resultados encontrados no cenário base da análise. Os parâmetros seguiram a mesma variação apresentada na análise de sensibilidade probabilística.

\section{Resultados}

\section{Análise de custo-efetividade}

Os resultados a seguir foram calculados para simular o custo de um paciente com migrânea crônica ao longo do horizonte de tempo avaliado.

\section{Resultados de custo e eficácia incremental}

A Tabela 6 apresenta os resultados de custo e eficácia da comparação da utilização da toxina onabotulínica A com "sem tratamento" para o tratamento de pacientes com migrânea crônica. Os resultados apontam reduções da ocorrência de dias e episódios de cefaleia ou migrânea a favor da toxina onabotulínica A, redução esta que varia de 44\% a 49\% em comparação ao "sem tratamento". Também se nota uma redução de 54\% a $60 \%$ nos custos médicos a favor da toxina onabotulínica A.

Calculou-se a razão de custo-efetividade incremental (RCEI) para cada desfecho, sendo que esta indica o custo relativo para a redução de um dia ou um episódio de cefaleia ou migrânea (Tabela 7).

Os resultados mostraram que o custo adicional proporcionado pelo uso da toxina onabotulínica A justifica o ganho clínico proporcionado para o paciente em termos de redução de um dia ou um episódio de cefaleia ou migrânea, para todos os horizontes de tempo avaliados. Observa-se que a maior RCEl encontrada foi de R\$208,85 por episódio de migrânea evitado. Esse valor é substancialmente baixo e permite a conclusão de que o uso da toxina onabotulínica $A$ é custo-efetiva em comparação à não profilaxia. 
Tabela 6. Resultados de custo e eficácia

\begin{tabular}{|c|c|c|c|}
\hline Desfechos & Toxina onabotulínica A & Sem tratamento & Incremental \\
\hline \multicolumn{4}{|l|}{24 semanas } \\
\hline Custo total & $\mathrm{R} \$ 9.506,03$ & $\mathrm{R} \$ 2.053,80$ & $\mathrm{R} \$ 7.452,23$ \\
\hline Tratamento & $\mathrm{R} \$ 8.645,54$ & $R \$ 0,00$ & $R \$ 8.645,54$ \\
\hline Consulta médica & $\mathrm{R} \$ 235,33$ & $R \$ 517,11$ & $-R \$ 281,78$ \\
\hline Visita à emergência & $R \$ 282,97$ & $\mathrm{R} \$ 681,22$ & $-R \$ 398,25$ \\
\hline Hospitalização & $\mathrm{R} \$ 342,19$ & $\mathrm{R} \$ 855,47$ & $-R \$ 513,28$ \\
\hline \multicolumn{4}{|l|}{ Eficácia } \\
\hline Dias com cefaleia & 66,38 & 118,80 & $-52,42$ \\
\hline Dias com cefaleia moderada/severa & 60,03 & 108,00 & $-47,97$ \\
\hline Episódios de cefaleia & 40,40 & 78,00 & $-37,60$ \\
\hline Dias com migrânea & 62,91 & 113,40 & $-50,49$ \\
\hline Episódios de migrânea & 37,52 & 73,20 & $-35,68$ \\
\hline \multicolumn{4}{|l|}{48 semanas } \\
\hline Custo total & $\mathrm{R} \$ 18.683,53$ & $\mathrm{R} \$ 4.107,60$ & $\mathrm{R} \$ 14.575,94$ \\
\hline Tratamento & $R \$ 16.962,56$ & $R \$ 0,00$ & $R \$ 16.962,56$ \\
\hline Consulta médica & $R \$ 470,66$ & $R \$ 1.034,22$ & $-R \$ 563,56$ \\
\hline Visita à emergência & $R \$ 565,93$ & $R \$ 1.362,43$ & $-R \$ 796,50$ \\
\hline Hospitalização & $\mathrm{R} \$ 684,38$ & $\mathrm{R} \$ 1.710,94$ & $-R \$ 1.026,57$ \\
\hline \multicolumn{4}{|l|}{ Eficácia } \\
\hline Dias com cefaleia & 132,76 & 237,60 & $-104,84$ \\
\hline Dias com cefaleia moderada/severa & 120,06 & 216,00 & $-95,94$ \\
\hline Episódios de cefaleia & 80,81 & 156,00 & $-75,19$ \\
\hline Dias com migrânea & 125,83 & 226,80 & $-100,97$ \\
\hline Episódios de migrânea & 75,04 & 146,40 & $-71,36$ \\
\hline \multicolumn{4}{|l|}{96 semanas } \\
\hline Custo total & $\mathrm{R} \$ 37.038,53$ & $\mathrm{R} \$ 8.215,19$ & $\mathrm{R} \$ 28.823,34$ \\
\hline Tratamento & $R \$ 33.596,58$ & $R \$ 0,00$ & $R \$ 33.596,58$ \\
\hline Consulta médica & $R \$ 941,32$ & $R \$ 2.068,44$ & $-R \$ 1.127,11$ \\
\hline Visita à emergência & $\mathrm{R} \$ 1.131,87$ & $\mathrm{R} \$ 2.724,87$ & $-R \$ 1.593,00$ \\
\hline Hospitalização & $R \$ 1.368,75$ & $R \$ 3.421,89$ & $-R \$ 2.053,13$ \\
\hline \multicolumn{4}{|l|}{ Eficácia } \\
\hline Dias com cefaleia & 265,51 & 475,20 & $-209,69$ \\
\hline Dias com cefaleia moderada/severa & 240,12 & 432,00 & $-191,88$ \\
\hline Episódios de cefaleia & 161,62 & 312,00 & $-150,38$ \\
\hline Dias com migrânea & 251,66 & 453,60 & $-201,94$ \\
\hline Episódios de migrânea & 150,07 & 292,80 & $-142,73$ \\
\hline
\end{tabular}

Tabela 7. Razão custo-efetividade incremental (RCEI)

\begin{tabular}{lccc}
\hline Custo por cada tipo de desfecho reduzido & $\begin{array}{c}\text { RCEI } \\
\text { 24 semanas }\end{array}$ & $\begin{array}{c}\text { RCEI } \\
\text { 48 semanas }\end{array}$ & RCEI semanas \\
\hline Dias com cefaleia & $R \$ 142,16$ & $R \$ 139,02$ & $R \$ 137,46$ \\
\hline Dias com cefaleia moderada/severa & $R \$ 155,35$ & $R \$ 151,92$ & $R \$ 150,21$ \\
\hline Episódios de cefaleia & $R \$ 198,22$ & $R \$ 193,85$ & $R \$ 191,66$ \\
\hline Dias com migrânea & $R \$ 147,61$ & $R \$ 144,36$ & $R \$ 142,73$ \\
\hline Episódios de migrânea & $R \$ 208,85$ & $R \$ 204,25$ & $R \$ 201,95$ \\
\hline
\end{tabular}




\section{Análise de sensibilidade}

\section{Análise de sensibilidade probabilística}

Para a análise de sensibilidade probabilística, avaliaram-se o horizonte de tempo de 24 semanas e o desfecho de redução de dias de migrânea. Assim, os desfechos apresentados que demonstram valores negativo $(<0)$ são considerados favoráveis ao tratamento com toxina onabotulínica $A$.

Os parâmetros dos valores basais foram variados de acordo com os desvios-padrão apresentados no estudo e os demais parâmetros foram variados em 10\% (Tabela 8).

Das 1.000 iterações, todas apresentaram incremento de custo, sendo que 93,7\% apresentaram redução de dias de migrânea a favor da toxina onabotulínica A (Figura 1).

\section{Análise de sensibilidade univariada}

Assim como na análise de sensibilidade probabilística, esta análise foi realizada considerando o horizonte temporal de 24 semanas e o desfecho de dias de migrânea (Tabela 9).
A variação dos parâmetros resultou em alterações do RCEl de $\mathrm{R} \$ 99,65$ a $\mathrm{R} \$ 284,60$, corroborando com os resultados encontrados no caso base (Figura 2).

\section{Conclusão}

Considerando-se a magnitude do benefício clínico e perfil de segurança favorável observados com o uso da toxina onabotulínica A durante o tratamento de pacientes com migrânea crônica, acredita-se que seu uso proporciona redução substancial do consumo de medicamentos e visitas médicas associada a uma baixa prevalência de eventos adversos.

Vale ressaltar que esses resultados podem estar subestimados, uma vez que custos indiretos, em que se evidenciam o impacto da migrânea e cefaleia na capacidade em levar uma vida normal e a consequente influência desse fator em sua capacidade produtiva, não foram contemplados na análise.

Pacientes com migrânea crônica possuem limitações significativas na qualidade de vida, apresentando, por exemplo,

Tabela 8. Parâmetros variados para a ASP

\begin{tabular}{|c|c|c|c|}
\hline Parâmetro & Distribuição & Base & DP \\
\hline Baseline toxina onabotulínica A - Dias com cefaleia & Gama & 19,90 & 3,68 \\
\hline Baseline toxina onabotulínica A - Dias com cefaleia moderada/severa & Gama & 18,10 & 4,12 \\
\hline Baseline toxina onabotulínica A - Episódios de cefaleia & Gama & 12,20 & 5,25 \\
\hline Baseline toxina onabotulínica tipo A - Dias com migrânea & Gama & 19,10 & 3,99 \\
\hline Baseline Botox toxina onabotulínica A - Episódios de migrânea & Gama & 11,40 & 5,02 \\
\hline Baseline sem tratamento - Dias com cefaleia & Gama & 19,80 & 3,68 \\
\hline Baseline sem tratamento - Dias com cefaleia moderada/severa & Gama & 18,00 & 4,25 \\
\hline Baseline sem tratamento - Episódios de cefaleia & Gama & 13,00 & 5,50 \\
\hline Baseline sem tratamento - Dias com migrânea & Gama & 18,90 & 4,05 \\
\hline Baseline sem tratamento - Episódios de migrânea & Gama & 12,20 & 5,42 \\
\hline Mudança toxina onabotulínica A - Dias com cefaleia & Gama & $-8,40$ & $-0,84$ \\
\hline Mudança toxina onabotulínica A - Dias com cefaleia moderada/severa & Gama & $-7,70$ & $-0,77$ \\
\hline Mudança toxina onabotulínica A - Episódios de cefaleia & Gama & $-5,20$ & $-0,52$ \\
\hline Mudança toxina onabotulínica A - Dias com migrânea & Gama & $-8,20$ & $-0,82$ \\
\hline Mudança toxina onabotulínica A - Episódios de migrânea & Gama & $-4,90$ & $-0,49$ \\
\hline Recursos (consulta médica) - Pré-tratamento & Gama & 1,67 & 0,17 \\
\hline Recursos (visita à emergência) - Pré-tratamento & Gama & 0,65 & 0,07 \\
\hline Recursos (hospitalização) - Pré-tratamento & Gama & 0,20 & 0,02 \\
\hline Recursos (consulta médica) - Pós-tratamento (toxina onabotulínica A) & Gama & 0,76 & 0,08 \\
\hline Recursos (visita à emergência) - Pós-tratamento (toxina onabotulínica A) & Gama & 0,27 & 0,03 \\
\hline Recursos (hospitalização) - Pós-tratamento (toxina onabotulínica A) & Gama & 0,08 & 0,01 \\
\hline Descontinuação (toxina onabotulínica A) & Beta & 0,04 & 0,00 \\
\hline Custos - Consulta médica & Gama & 309,65 & 30,96 \\
\hline Custos - Visita à emergência & Gama & 1048,03 & 104,80 \\
\hline Custos - Hospitalização & Gama & 4277,36 & 427,74 \\
\hline
\end{tabular}

ASP: Análise de sensibilidade probabilística. 


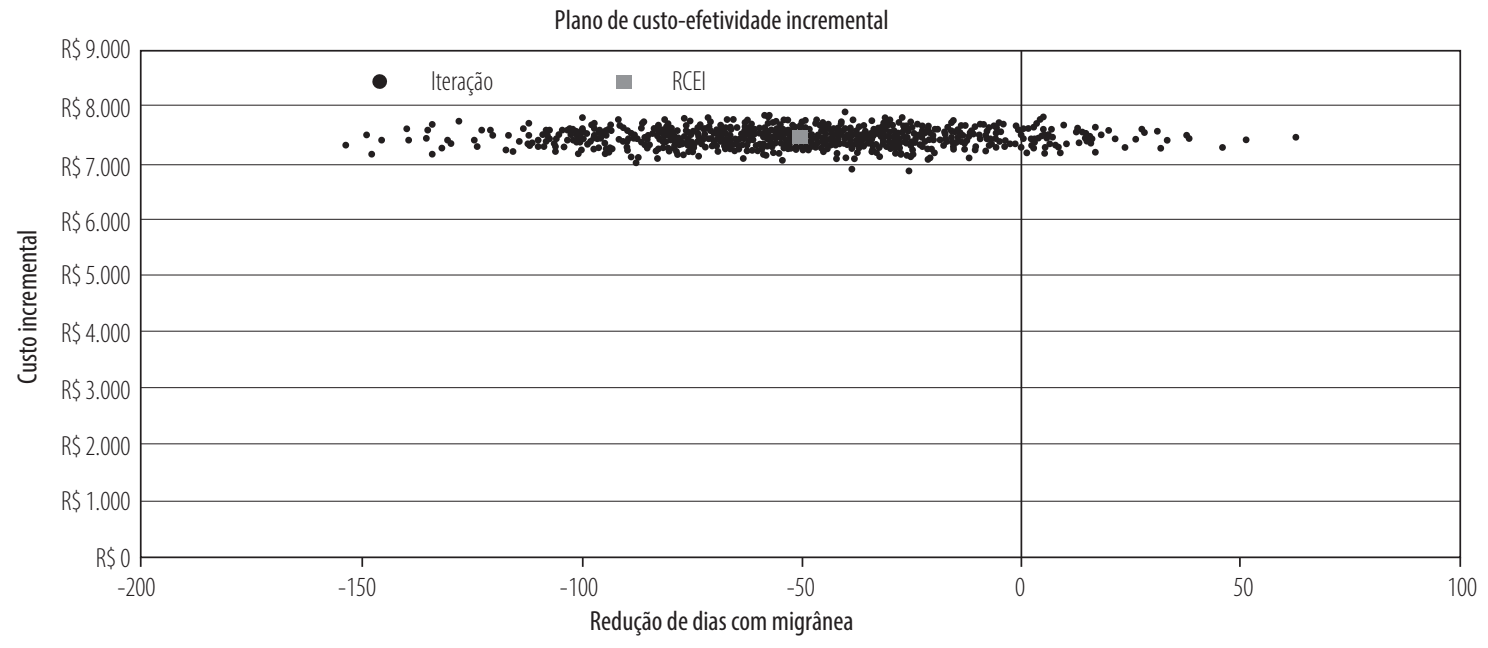

Figura 1. Plano de custo-efetividade incremental.

Tabela 9. Parâmetros variados na análise de sensibilidade univariada

\begin{tabular}{lccc}
\hline Parâmetros & Cenário base & Mínimo & Máximo \\
\hline Baseline sem tratamento - Dias com migrânea & 18,90 & 14,85 & 22,95 \\
\hline Baseline toxina onabotulínica A - Dias com migrânea & 19,10 & 15,11 & 23,09 \\
\hline Mudança toxina onabotulínica A - Dias com migrânea & $-8,20$ & $-7,38$ & 0,18 \\
\hline Recursos (Hospitalização) - Pré-tratamento & 0,20 & 0,59 & 0,22 \\
\hline Recursos (Visita à emergência) - Pré-tratamento & 0,65 & 1,50 & 0,72 \\
\hline Recursos (Consulta médica) - Pré-tratamento & 1,67 & $3.849,62$ & $4.705,09$ \\
\hline Custos - Hospitalização & $4.277,36$ & 4 \\
\hline
\end{tabular}

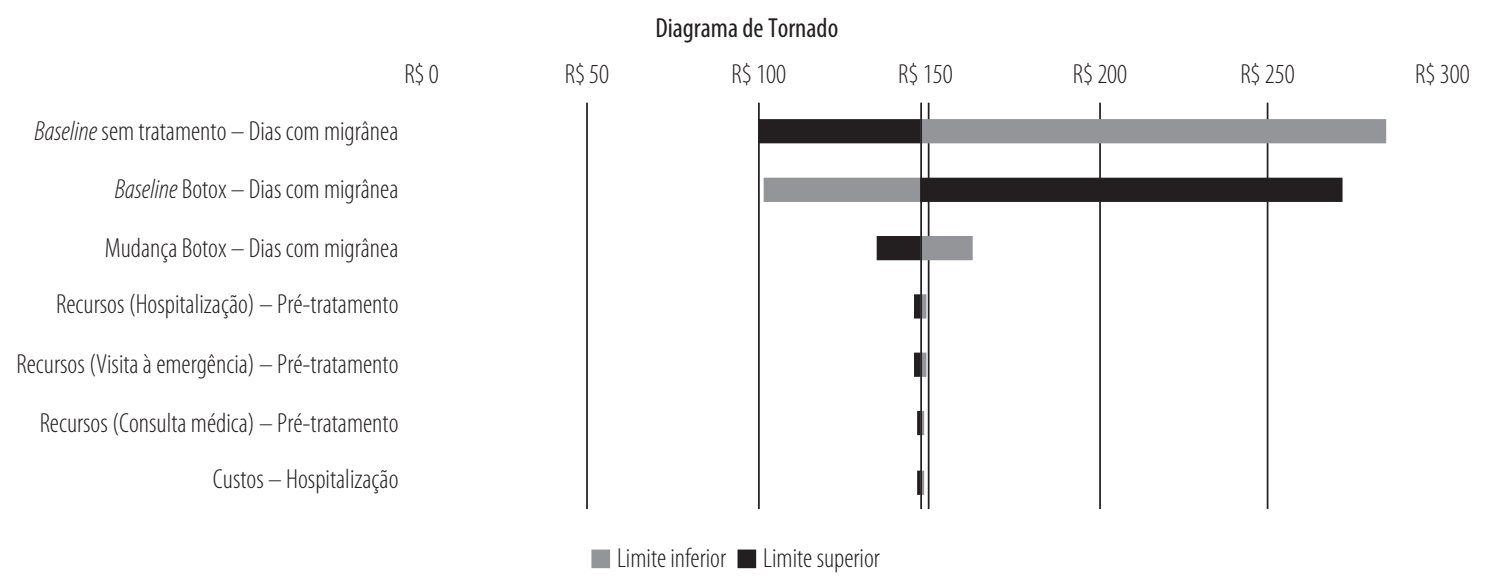

Figura 2. Diagrama de tornado.

elevada comorbidade com transtornos de humor e ansiedade (Breslau et al., 2001; Merikangas et al., 1990). Adicionalmente, também apresentam asma e dor musculoesquelética crônica (Terwindt et al., 2000). Comparados aos pacientes não migranosos e com outras condições crônicas, pacientes com migrânea relatam comprometimento no funcionamento físico, mental e social, particularmente naqueles com uma elevada frequência dos episódios (Terwindt et al., 2000). Em um estudo neuropsicológico sobre migrânea, foi constatado que os pacientes com a migrânea apresentam pior desempenho 
em tarefas que exigem habilidades de memória verbal, velocidades de processamento visual e função executiva, quando comparados a pessoas com ausência de cefaleia (Silva \& Teixeira, 2008).

Breslau et al. (2003) observaram uma relação bidirecional entre migrânea e depressão. A presença de uma das condições aumenta consideravelmente a chance de desenvolvimento da outra (Breslau et al., 2003). Em uma amostra populacional de indivíduos com migrânea, Lipton et al. (2000) avaliaram a influência da migrânea e depressão na qualidade de vida relacionada à saúde (QVRS). Em comparação com a população controle (ausência de cefaleia), os pacientes portadores de migrânea crônica apresentaram menor QVRS baseado no questionário SF-12 (Lipton et al., 2000). Além disso, foi visto que, quando comparados a outras condições crônicas, como osteoartrose, depressão e diabetes mellitus, os migranosos obtiveram as piores pontuações de qualidade de vida, juntamente com os portadores de depressão. Muitos prejudicam a sua decisão em relação a empregos e evitam situações sociais ou festas em que possam desencadear episódios de cefaleia, o que indica que a migrânea diminui significativamente a qualidade de vida não somente durante as crises, como também nos períodos em que a ansiedade, o medo e a incerteza contribuem para uma exclusão social (Bigal et al., 2000).

Vale ressaltar que a escolha da estrutura do modelo utilizada nesta avaliação econômica procurou seguir estrutura similar a já adotada em modelos desenvolvidos internacionalmente (Rothrock et al., 2014).

Além disso, foi realizada análise de sensibilidade probabilística e univariada para quantificar a incerteza envolvida nos resultados e identificar as variáveis que mais afetam essa incerteza, validando, dessa forma, os resultados encontrados e minimizando o impacto das limitações anteriormente expostas. As análises comprovaram os resultados encontrados no caso base.

Assim, o presente estudo é fundamental para, juntamente com as questões clínicas e sociais já abordadas, corroborar com dados econômicos que mostram que a toxina onabotulínica A é um tratamento eficaz, seguro e que promove redução substancial no consumo de medicamentos e visitas médicas e baixa prevalência de eventos adversos, sob a perspectiva do mercado privado de saúde do Brasil.

\section{Referências bibliográficas}

Agência Nacional de Vigilância Sanitária (Anvisa). Câmara de Regulação do Mercado de Medicamentos (CMED) [Internet]. 2016. Disponível em: http://portal.anvisa.gov.br/cmed. Acesso em: maio 2017.

Allergan INC. Botox ${ }^{\oplus}$. [Bula]. [Internet]. Brasília; 2015. Disponível em: http:// www.anvisa.gov.br/fila_bula/frmVisualizarBula.asp?pNuTransacao $=6716$ 462013\&pldAnexo=1742208. Acesso em: maio 2017.
American Academy of Neurology. Practice Guideline Update Summary: Botulinum Neurotoxin for the Treatment of Blepharospasm, Cervical Dystonia, Adult Spasticity, and Headache. Neurology. 2016;86(19):1818-26.

Associação Médica Brasileira (AMB). Classificação Brasileira Hierarquizada de Procedimentos Médicos - CBHPM. 2015

Aurora SK, Dodick DW, DeGryse RE, Turkel CC. OnabotulinumtoxinA for chronic migraine: efficacy and tolerability in patients who received all 5 treatment cycles in the PREEMPT clinical program. Headache. 2011:51(Suppl 1):61.

Aurora SK, Dodick DW, Turkel CC, DeGryse RE, Silberstein SD, Lipton RB, et al. OnabotulinumtoxinA for treatment of chronic migraine: results from the double-blind, randomized, placebo-controlled phase of the PREEMPT 1 trial. Cephalalgia. 2010;30(7):793-803.

Benseñor IM, Lotufo PA, Goulart AC, Menezes PR, Scazufca M. The prevalence of headache among elderly in a low-income area of São Paulo, Brazil. Cephalalgia. 2008;28(4):329-33.

Bigal ME, Fernandes LC, Moraes FA, Bordini CA, Speciali JG. Prevalência e impacto da migrânea em funcionários do Hospital das Clínicas da Faculdade de Medicina Ribeirão Preto - USP. Arq Neuropsiquiatr. 2000;58(2-B):431-6.

Breslau N, Lipton RB, Stewart WF, Schultz LR, Welch KMA. Comorbidity of migraine and depression: investigating potential etiology and prognosis. Neurology. 2003;60(8):1308-12.

Breslau N, Schultz LR, Stewart WF, Lipton R, Welch KM. Headache types and panic disorder: directionality and specificity. Neurology. 2001;56(3):350-4.

Bumb A, Seifert B, Wetzel S, Agosti R. Patients profiling for Botox ${ }^{\oplus}$ (onabotulinum toxin $A$ ) treatment for migraine: a look at white matter lesions in the MRI as a potential marker. Springerplus. 2013;2:377.

Diener HC, Dodick DW, Aurora SK, Turkel CC, DeGryse RE, Lipton RB, et al. OnabotulinumtoxinA for treatment of chronic migraine: Results from the double-blind, randomized, placebo-controlled phase of the PREEMPT 2 trial. Cephalagia. 2010;30(7):804-14.

Dodick DW, Turkel CC, DeGryse RE, Aurora SK, Silberstein SD, Lipton RB, et al. OnabotulinumtoxinA for treatment of chronic migraine: pooled results from the double-blind, randomized, placebo-controlled phases of the PREEMPT clinical program. Headache. 2010;50(6):921-36.

Felício AC, Bichuetti DB, Adolfo W. Epidemiology of Primary and TertiaryCare Center. 2006;64(October 2005):41-4.

Frampton JE. OnabotulinumtoxinA (BOTOX) A Review of its Use in the Prophylaxis of Headaches in Adults with Chronic Migraine. Drugs. 2012;72(6):825-45.

Giacomozzi AR, Vindas AP, da Silva Junior AA, Bordini CA, Buonanotte CF, Roesler CA, et al. Consenso latino-americano para as diretrizes de tratamento da migrânea crônica. Headache Med. 2012;3(4):150-61

Headache Classification Committee of the International Headache Society (IHS). The International Classification of Headache Disorders, 3rd edition (beta version). Cephalalgia. 2013;33(9):629-808.

Jackson JL, Kuriyama A, Hayashino Y. Botulinum toxin A for prophylactic treatment of migraine and tension headaches in adults: a meta-analysis JAMA. 2012;307(16):1736-45.

Krymchantowski AV, Ferreira P, Filho M. Atualização no tratamento profilático das enxaquecas. Arq Neuropsiquiatr. 1999;57(2-B):513-9.

Linsenmeyer TA. Use of botulinum toxin in individuals with neurogenic detrusor overactivity: state of the art review. J Spinal Cord Med. 2013;36(5):402-19.

Lipton RB, Hamelsky SW, Kolodner KB, Steiner TJ, Stewart WF. Migraine, quality of life, and depression: a population-based case-control study. Neurology. 2000;55(5):629-35. 
Lipton RB, Bigal ME, Diamond M, Freitag F, Reed ML, Stewart WF; AMPP Advisory Group. Migraine prevalence, disease burden, and the need for preventive therapy. Neurology. 2007;68(5):343-9.

Martins KM, Bordini CA, Bigal ME, Speciali JG. Migraine in the Elderly: A Comparison with Migraine in Young Adults. Headache. 2006;46(2):312-6.

Matthew N, Frishberg B, Gawel M, Dimitrova R, Gibson J, Turkel C. Botulinum toxin type $\left.A\left(B^{\circ}\right)^{\oplus}\right)$ for the prophylactic treatment of chronic daily headache: a randomized, double-blind, placebo-controlled trial. Headache. 2005;45(293-307):1126-37.

Medeiros FLM, Medeiros PLM, Silva WFS, Rodrigues JAR, Reis WLR. Tratamento profilático da migrânea. Migrâneas Cefaléias. 2008;11(1):33-41.

Merikangas KR, Angst J, Isler H. Migraine and psychopathology. Results of the Zurich cohort study of young adults. Arch Gen Psychiatry. 1990;47(9):849-53.

Ministério da Saúde (Brasil). Secretaria de Ciência, Tecnologia e Insumos Estratégicos. Departamento de Ciência e Tecnologia. Diretrizes metodológicas: Estudos de Avaliação Econômica de Tecnologias em Saúde. Brasília: Ministério da Saúde; 2009. 152 p.

Natoli JL, Manack A, Dean B, Butler Q, Turkel CC, Stovner L, et al. Global prevalence of chronic migraine: a systematic review. Cephalalgia. 2010;30(5):599-609.

Pahim LS, Menezes AMB, Lima R. Prevalência e fatores associados à enxaqueca na população adulta de Pelotas, RS. Rev Saúde Pública. 2006:40(4):692-8.

Queiroz LP, Peres MFP, Piovesan EJ, Kowacs F, Ciciarelli MC, Souza JA, et al. A nationwide population-based study of migraine in Brazil. Cephalalgia. 2009:29(6):642-9.
Robertson CE, Garza I. Critical analysis of the use of onabotulinumtoxinA (botulinum toxin type A) in migraine. Neuropsychiatr Dis Treat. 2012;8:35-48.

Rothrock JF, Bloudek LM, Houle TT, Andress-Rothrock D, Varon SF. Real-world economic impact of onabotulinumtoxina in patients with chronic migraine. Headache. 2014;54(10):1565-73.

Silva M, Teixeira A. Neuropsicologia das cefaléias. Migrâneas Cefaléias. 2008;11(2):114-7.

Silberstein S, Stark S, Lucas S, Christie S, Degryse R, Turkel C. Botulinum toxin type A for the prophylactic treatment of chronic daily headache: a randomized, double-blind, placebo-controlled trial. Mayo Clin Proc. 2005;80(9):1126-37.

Sociedade Brasileira de Medicina de Família e Comunidade, Associação Brasileira de Medicina Física e Reabilitação, Academia Brasileira de Neurologia. Projeto Diretrizes: Cefaleias em Adultos na Atenção Primária à Saúde: Diagnóstico e Tratamento. São Paulo: AMB; CFM; 2009. p. 1-14.

Stovner LJ, Hagen K, Jensen R, Katsarava Z, Lipton R, Scher A, et al. The global burden of headache: a documentation of headache prevalence and disability worldwide. Cephalalgia. 2007;27(3):193-210.

Terwindt GM, Ferrari MD, Tijhuis M, Groenen SM, Picavet HS, Launer LJ. The impact of migraine on quality of life in the general population: the GEM study. Neurology. 2000;55(5):62-9.

World Health Organization (WHO). Atlas of headache disorders and resources in the world 2011 [Internet]. 2011. Disponível em: http://www. who.int/mental_health/management/atlas_headache_disorders/en/. Acesso em: maio 2017. 